\title{
Integração de um agente conversacional no processo de ensino e aprendizagem utilizando as teorias MCE e MMEEBB para a retenção do conhecimento
}

\author{
Mislene D. Silva ${ }^{1}$, Sara L. de Melo ${ }^{1}$, Mirian C. Silva ${ }^{1}$, Luciano V. Lima ${ }^{1}$ \\ ${ }^{1}$ Departamento de Engenharia Elétrica \\ Universidade Federal de Uberlândia - UFU \\ Uberlândia - MG - Brasil \\ \{mislenedalila,saraluziamelo,miriancamila0,lucianovieiralimaster\}@ gmail.com
}

\begin{abstract}
This is paper proposes the development of a conversational agent in a teaching computational environment. Thus, for the development of the agent, theories of Structured Knowledge Maps and Methods of Effective Exponential Storage in the Binary Base were used in order to separate the concepts and knowledge of the student, as well as to ensure the memorization of the learned content. In addition, the agent was integrated into a virtual learning environment for interaction with students, which resulted in good results in the evaluation process.
\end{abstract}

Resumo. Este trabalho propõe o desenvolvimento de um agente conversacional em um ambiente computacional de ensino. Assim, para o desenvolvimento do agente foram utilizadas as teorias Mapas de Conhecimentos Estruturados (MCE) e Métodos de Memorização Exponencial Efetivo na Base Binária (MMEEBB) com a finalidade de separar os conceitos e conhecimentos do estudante, bem como garantir a memorização do conteúdo aprendido. Além disso, o agente foi integrado em um ambiente virtual de aprendizagem para interação com os estudantes o que resultou em bons resultados no processo avaliativo.

\section{Introdução}

Atualmente muitas atividades em todos os setores estão sendo automatizados, processos de atendimentos, suportes entre outros. Este cenário está cada vez mais próximo do ambiente educacional. Fato este já visualizado em processos de renegociação de dívidas de alunos e atendimento.

Muitas tecnologias são aplicadas no processo de ensino e aprendizagem, mas nem sempre essas ferramentas são efetivas e de fato proporcionam o aprendizado e, consequentemente, a satisfação do aluno.

Um agente conversacional ou chatbot é um software que interpreta e responde as declarações feitas por usuários, é o processo de interação com a máquina através de linguagem natural, ou seja, a máquina precisa fornecer ao indivíduo respostas informativas e manter um diálogo natural com uma similaridade ao ser humano [Ferreira, Tânia, et al.,2018]. Sendo assim, em um ambiente computacional de aprendizagem, espera-se de um agente conversacional a naturalidade de um diálogo 
VII Congresso Brasileiro de Informática na Educação (CBIE 2018)

Anais do XXIX Simpósio Brasileiro de Informática na Educação (SBIE 2018)

mais próximo do natural, ou seja, similar a de um humano. São esses detalhes que sobressaem na implantação de bots em qualquer setor. O trabalho de [Júnior, Cleon Pereira, et al.,2017] efetuaram uma revisão sistemática sobre a utilização de agentes conversacionais com ontologias para o ensino- aprendizagem. Nesta pesquisa foram levantadas questões de pesquisas sobre requisitos funcionais e não funcionais para $o$ desenvolvimento de um agente conversacional.

Levando em consideração a habilidade de um bot e acessibilidade do mesmo, nesta pesquisa propõe o desenvolvimento de um agente conversacional, utilizando o método de retenção do conhecimento na base binária (MMEEBB) [Ferreira, Daniela Carvalho Monteiro, et al.,2014] que auxilia na memorização a fim de engajar o aluno e, alcançar bons resultados no processo de aprendizado. Para a formação dos objetos de aprendizagem (OA) o bot levou em consideração a teoria de Mapas de Conhecimentos Estruturados (MCE) [Ferreira, Daniela Carvalho Monteiro, et al.,2014].

Desse modo, o agente conversacional desenvolvido foi implantado em uma plataforma interativa para alunos de graduação de uma instituição superior de ensino, com intuito de auxiliar cada estudante de maneira personalizada no processo de aprendizagem e na retenção do conhecimento. Em seguida, efetuou-se uma análise com 56 alunos dos cursos de Administração e Direito, na disciplina Cultura e Sociedade. Os alunos que tiveram contato com o agente conversacional alcançaram resultados positivos quanto à habilidade do bot para sanar dúvidas e disponibilizar conteúdos.

\section{Trabalhos Correlatos}

Agentes conversacionais estão sendo utilizados em vários setores, como: atendimento a clientes, para gerenciamento de ações através de dispositivos móveis, games e também são alvos da educação nas diversas áreas desde os atendimentos acadêmicos como financeiro, matrículas a disponibilização de materiais, bem como integração em ambientes computacionais de ensino para auxílio de determinado conteúdo.

[Moraes e Wilkens 2008] exibe em seu trabalho a elaboração de um agente conversacional emocional que investiga a comunicação sobre as emoções e interpreta o modelo de personalidade Big-Five. Além disso, efetuou-se uma análise das consequências das emoções na área de interação. Para processar a análise do sistema conversacional, foi empregado o framework chatterbean, que possui algumas limitações como, não há suporte a HTML e as tags são acondicionadas de forma recursiva. No sistema emocional há a interpretação das emoções, personalidade, instintos e percepções internas do agente. Assim, são armazenados a emoção e o valor de cada emoção do estudante, bem como seus traços de personalidade.

No trabalho de [Ong e Soriano, 2016] foi retratado mecanismos para integração de um agente tutor conversacional em um bate-papo com estudantes filipinos de faixa etária entre 8-10 anos de idade no momento em que eles efetivavam uma avaliação de leitura e se sentiam negativos. Os agentes de conversação podem atrair os alunos de diversas formas, como, através de texto, fala, gestos com as mãos, expressões faciais, olhar nos olhos e outros tipos de linguagem corporal. Os autores salientam que o estado emocional interfere no desempenho do aprendizado. 
VII Congresso Brasileiro de Informática na Educação (CBIE 2018)

Anais do XXIX Simpósio Brasileiro de Informática na Educação (SBIE 2018)

Em [Ferreira, Tânia, et al.,2018] apresentou estudos sobre a execução de um agente conversacional chamado Spike composto a um ambiente virtual de aprendizagem (AVA) para suplementar no aprendizado dos estudantes. A técnica de inteligência artificial escolhida para a criação do agente foi a Alice que contém a arquitetura mais íntegra, que usa a linguagem de marcação AIML para diferenciar as bases de conhecimento. O agente conversacional foi integrado ao Moodle na Universidade de Cruz Alta para melhorar a experiência de aprendizagem dos alunos, tornando o ambiente mais amigável e dinâmico.

A seguir são descritos a metodologia e os métodos utilizados para o agente conversacional proposto neste trabalho, bem como a análise dos resultados obtidos por meio da interação com estudantes reais.

\section{O ambiente de aprendizagem e o Agente Conversacional}

O agente conversacional desenvolvido nesse trabalho é denominado LINA, o qual é responsável por manter uma interação com os estudantes, bem como selecionar os objetos de aprendizagem de acordo com a teoria Métodos de Memorização Exponencial Efetivo na Base Binária (MMEEBB), que determina um Intervalo de Reforço de Aprendizagem - IRA para o aluno memorizar o conhecimento e ser útil em momentos de necessidade. O conteúdo da disciplina Cultura e Sociedade foram divididos em 16 temáticas de acordo com a teoria utilizada.

Para seleção do material de ensino enviado para os alunos foi desenvolvido pelo professor da disciplina na instituição, seguindo a teoria Mapas de Conhecimento Estruturado - MCE, de acordo com essa teoria com intuito de evitar a sobrecarga cognitiva, determina para cada aluno um raio de conhecimento, onde são separados os pré-requisitos de conhecimento em relação ao conteúdo de determinado módulo do curso. Desse modo, para a interação do agente LINA com o estudante o agente leva em consideração as dúvidas de cada aluno para em seguida oferecer um objeto de aprendizagem de acordo com essas dúvidas, bem como auxilia-lo na memorização do conhecimento.

O ambiente de aprendizagem estabelecido para realizar a conversação entre os agentes foi através da rede social Facebook e do aplicativo Telegram. A escolha do Facebook foi principalmente pelo fato de $80 \%$ dos alunos estarem cadastrados na rede social e o link disponível no portal acadêmico (ambiente virtual de aprendizagem da instituição).

A plataforma utilizada para armazenar as interações do bot foi o DialogFlow. A ferramenta DialogFlow é um upgrade da api.ai desenvolvida pelo Google essas ferramentas possibilitam construir interfaces de conversação para bots, aplicativos e dispositivos [DialogFlow, 2018]. No ambiente da plataforma DialogFlow é necessário o entendimento de dois conceitos muito importantes para construção de bots, que são as entidades e as intenções.

As intenções (intents) são classificadas como as intenções do usuário, então nesse processo foi o momento de considerar o que os alunos poderiam fazer no ambiente do bot. Escolher a intenção correta para a entrada de um usuário é o primeiro passo para fornecer uma resposta útil. Por exemplo, na frase de treinamento do bot 
VII Congresso Brasileiro de Informática na Educação (CBIE 2018)

Anais do XXIX Simpósio Brasileiro de Informática na Educação (SBIE 2018)

"obrigado/ obrigada", quando o usuário digitar essa informação automaticamente a resposta do bot será: "De nada".

Outra intenção adicionada na plataforma foi para identificar o período em que o aluno está matriculado, foram cadastrados 10 períodos letivos, no momento que aluno responde o período a mensagem retornada, pode ser visualizada na Figura 01.

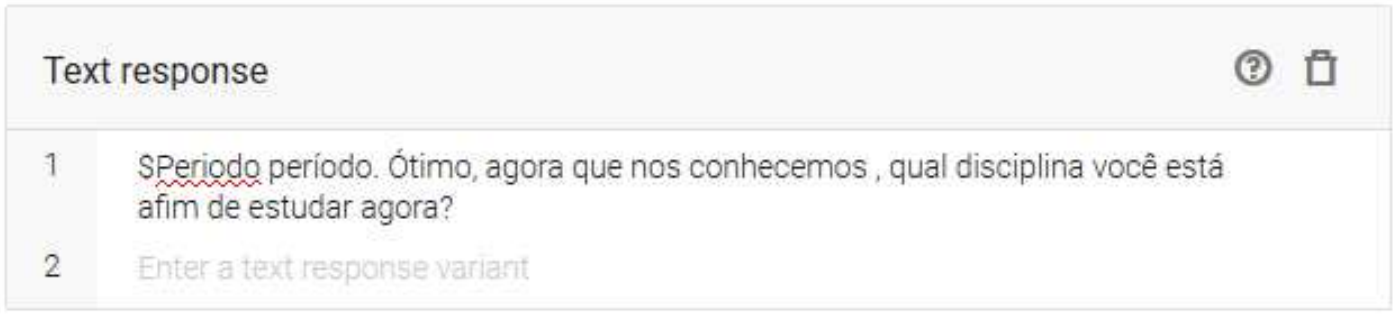

Figura 01 - Resposta cadastrada no ambiente DialogFlow. Fonte: PrintScreen DialogFlow, 2018.

Na Figura 01, você pode observar através do texto "\$Periodo" que é uma variável no início da resposta. Essa variável é o retorno da resposta do aluno, em relação ao qual o período que o mesmo está cursando.

As entidades representam um termo ou objeto na entrada do usuário que fornece esclarecimento ou contexto específico para uma determinada intenção. As intents representam verbo, algo que o indivíduo deseja fazer, já as entidades representa substantivos, ou seja, o contexto para a ação. No ambiente do dialogflow você pode inserir vários sinônimos que pode ser digitado pelo usuário. Trabalhando no mesmo contexto do exemplo anterior, os períodos letivos, as entidades cadastradas para período que possivelmente podem ser as entradas do usuário pode ser visualizada na Figura 02.

\begin{tabular}{|l|l|}
\hline Periodo & SAVE \\
\hline & \\
\hline Define synonyms 1 & Allow automated expansion \\
\hline segundo & segundo, $2^{\circ}, 2$ \\
\hline sexto & sexto, $6^{\circ}, 6$ \\
\hline quarto & quarto, $4^{\circ}, 4$ \\
\hline oitavo & oitavo, $8^{\circ}, 8$ \\
\hline decimo & decimo, $1^{\circ}, 10$ \\
\hline primeiro & primeiro, $1,1^{\circ}$ \\
\hline terceiro & terceiro, $3,3^{\circ}$ \\
\hline quinto & quinto, $5,5^{\circ}$ \\
\hline setimo & setimo, $7,7^{\circ}$ \\
\hline nono & nono, $9,9^{\circ}$ \\
\hline
\end{tabular}

Figura 02 - Sinônimos cadastrados para a entidade período. Fonte: PrintScreen DialogFlow, 2018. 
VII Congresso Brasileiro de Informática na Educação (CBIE 2018)

Anais do XXIX Simpósio Brasileiro de Informática na Educação (SBIE 2018)

Observa-se que são adicionadas várias possibilidades de entradas em relação à informação do período letivo que o aluno está cursando. Portanto se deseja capturar informações ou executar ações do usuário, utiliza-se uma intenção. Mas, se o objetivo é capturar informações que podem afetar a maneira como se responde uma solicitação ou ação, deve se utilizar entidades.

\subsection{A aplicação}

Para utilizar o bot desenvolvido para auxiliar os alunos nos estudos da disciplina de Cultura e Sociedade, deve se realizar o seguinte processo. No Facebook o aluno precisa buscar pela página "Virei Nerd". Na página foi criado um avatar para interagir com os alunos, chamada de LINA.

Na Figura 03, pode se visualizar a página inicial do Virei Nerd e a imagem da Lina, personagem que interage com os alunos.

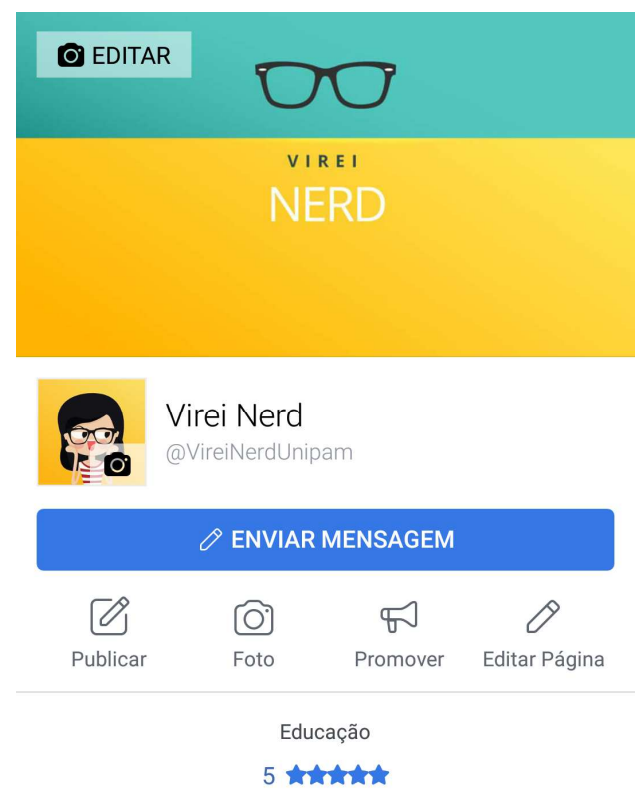

Figura 03 - Página Inicial Virei Nerd. Fonte: PrintScreen Facebook, 2018.

No Facebook para um chatbot iniciar um diálogo com o aluno a iniciativa deve partir do aluno. Na Figura 04 pode se visualizar como foi desenvolvido o primeiro diálogo com os alunos. 
VII Congresso Brasileiro de Informática na Educação (CBIE 2018)

Anais do XXIX Simpósio Brasileiro de Informática na Educação (SBIE 2018)

Quero participar da revisão

Oil E aí, beleza? Eu sou a Lina, e estou aqui para resolver

seus problemas kkkk

Para começarmos, preciso confirmar algumas informações,

ok?

Você está cursando a disciplina de Cultura e Sociedade

neste semestre?

$\operatorname{Sim}$

Cara, para começarmos preciso do seu usuário, aquele que você acessa o Portal Acadêmico... Mas calma aí, não mande a senha, heim...hahaha

Figura 04 - Diálogo Inicial com o aluno. Fonte: PrintScreen, página Virei Nerd, 2018.

Inicialmente o aluno interagiu com a Lina, com um "Quero participar da revisão", posteriormente a Lina realiza algumas perguntas para o aluno. Para identificar se está cursando a disciplina de Cultura e Sociedade no semestre. Solicita também o usuário do aluno, que é o seu número de matrícula, para validar se de fato o aluno está matriculado e cursando a disciplina de Cultura e Sociedade na instituição.

Se as informações do aluno estiverem corretas a Lina inicia um diálogo com aluno informando como será o processo de estudo com ela. Na Figura 05, pode se observar a conversa da Lina com o aluno.

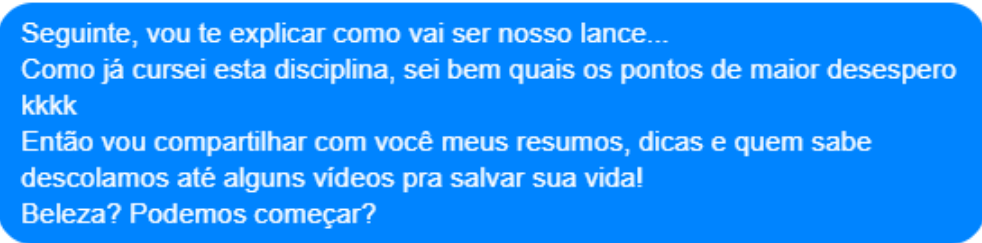

$4 / 6 / 201723: 56$

Figura 05 - Diálogo explicando como será o compartilhamento do material. Fonte: PrintScreen, página Virei Nerd, 2018.

Pode se visualizar através do dialogo da Lina com o aluno que o vocabulário utilizado nas conversas não é nada formal. Essa estratégia foi definida principalmente para Lina tornar-se uma amiga do aluno e está mais próximo do que ocorre no seu dia a dia. Para se chegar nesse resultado foi realizada uma consulta no banco de dados sobre faixa etária de idade dos participantes e elas prevaleceram entre 17 e 23 anos.

Feita a apresentação da Lina e a confirmação do aluno, inicia-se o processo de envio de conteúdos para o aluno. $\mathrm{O}$ envio como citado anteriormente seguiu o intervalo 
VII Congresso Brasileiro de Informática na Educação (CBIE 2018)

Anais do XXIX Simpósio Brasileiro de Informática na Educação (SBIE 2018)

da taxa IRA, com o espaçamento de $2^{\mathrm{n}}$ de notificações, seguindo o Método de Memorização Exponencial Efetivo na Base Binária - MMEEBB.

Na Figura 06, tem se uma interação da Lina enviando materiais para o aluno. A classificação dos materiais para envio foi definida seguindo o MCE, com informações resumidas e focadas em um conhecimento, evitando a sobrecarga cognitiva e a não memorização do conhecimento por parte do aluno. Na Figura 06, pode se visualizar ainda, através da resposta do aluno a satisfação do mesmo em receber os materiais resumidos para estudo.

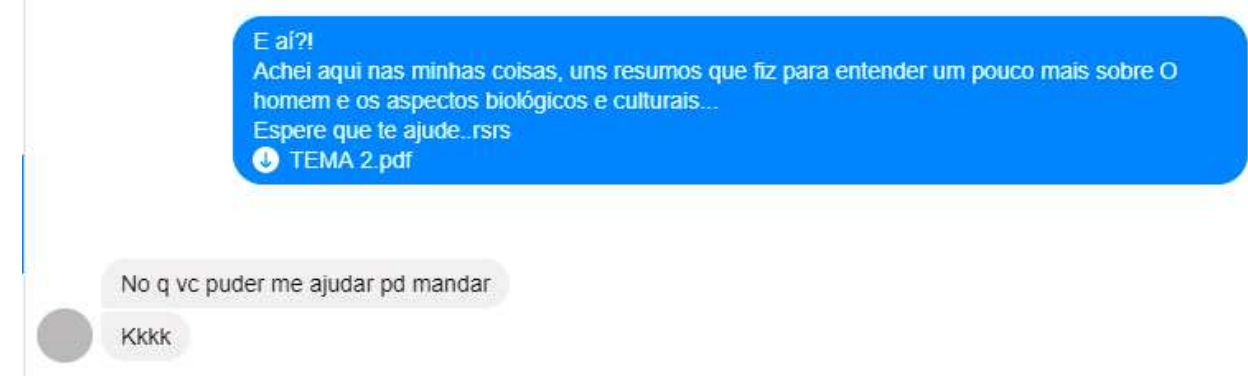

Figura 06 - Envio de conteúdo para o aluno. Fonte: PrintScreen, página Virei
Nerd, 2018.

Ao final do processo de interações a Lina recebeu comentários sendo parabenizada pelo o apoio nos estudos. Na Figura 07 pode se visualizar o depoimento de dois alunos em relação à ajuda que a Lina deu no decorrer dos estudos para as provas finais da disciplina de Cultura e Sociedade.

A prova colegiada e a AVIN citadas nos textos da Figura 07 são referentes às provas finais que os alunos cursam na disciplina de Cultura e Sociedade e devem fazer ao final do semestre. Os comentários mostram que o aluno foi com mais segurança para fazer a prova, pois foi possível ele memorizar informações "palavras" importantes para execução da prova. E no outro comentário mostra um resultado muito positivo com nota 18 na prova colegiada que totalizava 20.

\section{$28 \% 12017.20: 24$}

Foi muuito bom os conteudos, dar uma revisada nas materias que foi vista e propostas para a colegiada e a avin. Deu para esciarecer as duvidas e de formas simples e rápida guardar plalaras que tenho certeza que ajudar na hora de fazer as provas

\footnotetext{
Tirel 18 na colegiada

Errel apenas uma questão
}

$1 / 7 / 201714.20$

Figura 07 - Comentários de Agradecimento dos alunos. Fonte: PrintScreen, página Virei Nerd, 2018. 
VII Congresso Brasileiro de Informática na Educação (CBIE 2018)

Anais do XXIX Simpósio Brasileiro de Informática na Educação (SBIE 2018)

Na última interação com o aluno foi enviado um formulário para o mesmo para coletar informações sobre a satisfação do método utilizado para estudar através do bot. Nesse formulário as seguintes questões foram levantadas:

a) O melhor horário para o aluno receber notificações da Lina?

b) Quais os dias da semana gostariam de receber notificações?

c) Com qual frequência gostaria de receber conteúdos da Lina?

d) A nota que o aluno atribuía aos vídeos, infográficos e resumos?

e) A preferência de ambiente se seria um bate papo com a Lina o ou material disponibilização no Portal Acadêmico?

f) De 1 a 10, quanto o material contribui para sua aprendizagem?

g) De 1 a 10, quanto você indicaria o Virei Nerd para um colega?

h) $\mathrm{O}$ que você achou da experiência de estudos com a Lina?

Alguns dos resultados foram:

No total a página obteve 250 curtidas, 56 alunos participantes e mais de 156 mensagens recebidas. Na pergunta de 1 a 10, quanto você indicaria a página Virei Nerd para um colega?

Tabela 01. Indicação Virei Nerd.

\begin{tabular}{|c|c|}
\hline Quantidade de Pessoas & Nota \\
\hline 49 & 10 \\
\hline 4 & 9 \\
\hline 3 & 8 \\
\hline
\end{tabular}

O resultado como pode se observar na Tabela 01 , foi que 49 pessoas avaliaram com nota 10, 4 com e 3 com 8 . Na pergunta aplicada de quanto o material contribui para sua aprendizagem? O resultado pode ser visualizado na Tabela 02 , demostrando um resultado muito satisfatório da produção do material e a utilização do bot. Alcançando mais de $70 \%$ de aceitação do material disponibilizado.

Tabela 02 - Indicação Virei Nerd.

\begin{tabular}{|c|c|}
\hline Quantidade de Pessoas & Nota \\
\hline 39 & 10 \\
\hline 6 & 9 \\
\hline 7 & 8 \\
\hline 4 & 7 \\
\hline
\end{tabular}

Outro resultado positivo com o uso do bot foi se o aluno preferia que disponibilizassem o material através do bate-papo com a Lina ou através do Portal Acadêmico. As respostas foram $80 \%$ a favor da utilização da Lina no envio do conteúdo da disciplina.

\section{Considerações Finais}

Os testes realizados em uma população de 56 alunos entre 17 e 23 anos de idade, produziram resultados satisfatórios quanto à utilização do bot para disponibilização de 
VII Congresso Brasileiro de Informática na Educação (CBIE 2018)

Anais do XXIX Simpósio Brasileiro de Informática na Educação (SBIE 2018)

materiais resumidos para os alunos que cursavam a disciplina de Cultura e Sociedade. Fatos comprovados nas respostas dos alunos se preferiam que uma agente conversacional interagisse com ele enviando materiais e tirando dúvidas ou eles preferiam acessar diretamente o portal para baixar o material, o resultado foi que mais de $80 \%$ são a favor do uso do agente.

Resultado se deu provavelmente pela flexibilidade do discurso, simpatia do agente e também pela organização do material enviado pelo bot. Como citado anteriormente o mesmo seguiu as regras do MCE para produção o que não deixa produzir a sobrecarga cognitiva, ou seja, o excesso de conhecimento em um único raio de conhecimento.

Com a diversificação das mídias enviadas para os alunos observou-se que os mesmos possuem preferência por uma determinada mídia para estudo que são os vídeos, na pergunta sobre qual nota o aluno atribuía as mídias do tipo texto resumido, vídeo e infográficos, $90 \%$ dos alunos optaram pelo estudo através dos vídeos.

Portanto a implantação do bot para enviar materiais resumidos para os alunos foi bem positiva e os resultados mostraram bastantes aceitáveis. Além disso, a disponibilização do bot para novas disciplinas também está sendo providenciada.

\section{Referências}

DialogFlow. (2018) "Build natural and rich conversational experiences", https://dialogflow.com/,June.

Ferreira, Tânia \& Mariotto Mozzaquatro, Patrícia \& Rodovia, Municipal \& Jacoob, Della \& Benito Méa, Parada \& - Cruz, Alta \& , Rs \& , Brasil. (2018) "Spike: um agente conversacional integrado ao Ambiente Virtual de Aprendizagem Moodle".

Ferreira, Daniela Carvalho Monteiro, Lima, L. V., Camargo, J. H., Schiovato, N. S. C. (2014) "Mapas de Conhecimento Estruturado: proposta de uma nova abordagem metodológica de ensino e aprendizagem". Educere et Educare (versão eletrônica), v. 9,p.505-514.

Júnior, Cleon Pereira, et al. (2017) "Uso de Ontologias para Agentes Conversacionais no Contexto de Ensino-Aprendizagem: Uma Revisão Sistemática da Literatura." Brazilian Symposium on Computers in Education (Simpósio Brasileiro de Informática na Educação-SBIE). Vol. 28. No. 1.

Moraes, M. C.; Wilkens, Rodrigo. (2008) "Um Agente Conversacional Emocional". Hífen (Uruguaiana), $\quad$ v. $32, \quad$ p. $107-114$.

Ong E. C. J., Soriano Z. C. (2016) “A Conversational Agent to Shift Students' Affect State". In: Baldoni M. et al. (eds) Principles and Practice of Multi-Agent Systems. CMNA 2015, IWEC 2015, IWEC 2014. Lecture Notes in Computer Science, vol 9935. Springer, Cham. 